\title{
Bistable diode action in left-handed periodic structures
}

\author{
Michael W. Feise, Ilya V. Shadrivov, and Yuri S. Kivshar \\ Nonlinear Physics Centre and Centre for Ultra-High Bandwidth Devices for Optical Systems (CUDOS), Research School of Physical \\ Sciences and Engineering, Australian National University, Canberra, Australian Capital Territory 0200, Australia
}

(Received 1 December 2004; published 23 March 2005)

\begin{abstract}
We study nonlinear transmission of an asymmetric multilayer structure created by alternating slabs of two materials with positive and negative refractive indices. We demonstrate that such a structure exhibits passive spatially nonreciprocal transmission of electromagnetic waves, the analog of the electronic diode. We study the properties of this left-handed diode and confirm its highly nonreciprocal and bistable transmittance by employing direct simulations.
\end{abstract}

DOI: 10.1103/PhysRevE.71.037602

PACS number(s): 42.70.Qs, 41.20.Jb, 78.20.-e

An all-optical diode is a spatially nonreciprocal device that allows unidirectional propagation of a signal at a given wavelength. In the ideal case, the diode transmission is $100 \%$ in the forward propagation, whereas it is much smaller or vanishes for backward (opposite) propagation, yielding a unitary contrast. The possibility of achieving highly nonreciprocal transmission through a passive, nonlinear device is not only an interesting concept, but also useful for many applications such as optical isolation and all-optical processing. Such unidirectional action was demonstrated for several asymmetric structures and different nonlinear materials $[1-7]$.

In this paper, we discuss a spatially nonreciprocal device based on an asymmetric multilayered structure created by alternating slabs of two materials, conventional and left handed (LH); the latter is a material with both negative electric permittivity and negative magnetic permeability which results in an effective negative refractive index [8]. Such multilayered structures are a special class of structures consisting of a sequence of flat lenses that provide optical cancellation of the conventional, or right-handed $(\mathrm{RH})$ layers leading to either enhanced or suppressed transmission [9-11].

We employ the general idea of obtaining diode action by making the structure both asymmetric and nonlinear. Also, we consider a periodic superlattice, which is expected to possess resonant properties, and thus enhance nonlinear effects. We consider an asymmetric superlattice consisting of three stacks with four LH/RH double layers each [see Figs. 1(a) and $1(\mathrm{~b})]$. We assume that in the nonlinear regime, a dielectric defect layer with Kerr-type nonlinear response is inserted between stacks 1 and 2, as shown in Fig. 1(b). We study wave transmission in both linear and nonlinear regimes by using the transfer-matrix method (TMM) [12], and also by employing direct numerical simulations based on the pseudospectral time-domain (PSTD) method [13]. The transfer matrix of the system analytically relates the electromagnetic fields on either side of an element [12]. Complex structures can be built up out of simple elements through multiplication of the respective matrices. Thin nonlinear elements can be modeled, e.g., as $\delta$ functions [11,14].

In the PSTD method, the Maxwell equations are discretized in time and space. The spatial derivatives are approximated using discrete Fourier transforms and the tempo- ral derivatives using central differences. From this, one derives update equations for the different fields and by iteration propagates the fields through time. The problem of the inherent periodicity of the Fourier transform can be removed through the use of perfectly-matched-layer absorbing boundary conditions $[13,15]$. The PSTD method samples all field components at the same location in the unit cell, which is advantageous when both the electric permittivity and the magnetic permeability vary with position [16]. An instantaneous Kerr nonlinear material is included by directly solving the cubic equation for the intensity [17] at each iteration.

We model the LH material as having Lorentzian frequency dependence in both electric permittivity $\varepsilon_{r}$ and magnetic permeability $\mu_{r}$,

$$
\begin{gathered}
\varepsilon_{r}(\omega)=1+\frac{\omega_{p e}^{2}}{\omega_{1 e}^{2}-\omega^{2}-i \gamma_{e} \omega}, \\
\mu_{r}(\omega)=1+\frac{\omega_{p m}^{2}}{\omega_{1 m}^{2}-\omega^{2}-i \gamma_{m} \omega} .
\end{gathered}
$$

Here, $\omega_{p e}, \omega_{p m}$ are the corresponding plasma frequencies, $\omega_{1 e}, \omega_{1 m}$ are the resonance frequencies, and $\gamma_{e}, \gamma_{m}$ are ab-
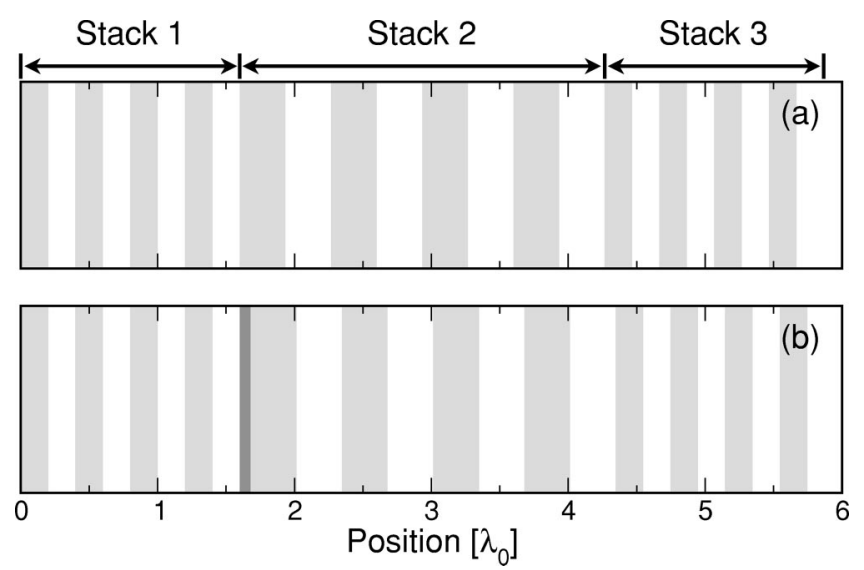

FIG. 1. Schematic of the LH/RH superlattice for transmission in (a) linear regime and (b) nonlinear diode regime. Layers of $\mathrm{LH}$ material are shown in light gray. The dielectric defect layer is shown in dark gray. 


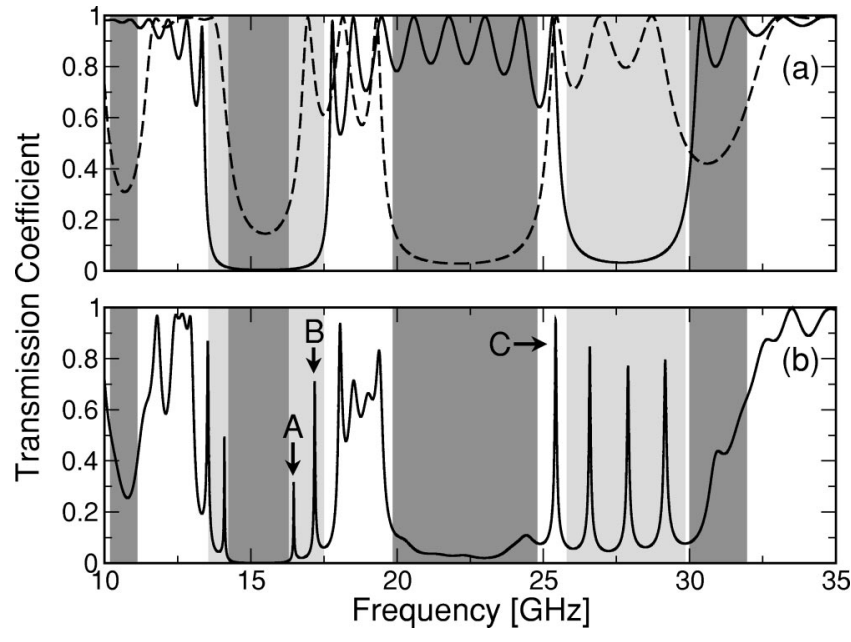

FIG. 2. Transmission spectrum of the structure and its components in the linear regime. (a) Stack of eight periods with individual layer thickness $\lambda_{0} / 3$ (solid) [stack 1 and stack 3 in Fig. 1(a)] and a stack of four periods with individual layer thickness $\lambda_{0} / 5$ (dashed) [stack 2 in Fig. 1(a)]. (b) Combination of these stacks when arranged as in Fig. 1(a). The shading indicates the location of the band gaps for stack 1 (light gray) and stack 2 (dark gray) in the limit of an infinite number of periods.

sorption parameters. We use $\omega_{p e}=1.1543 \times 10^{11} \mathrm{~s}^{-1}, \omega_{p m}$ $=1.6324 \times 10^{11} \mathrm{~s}^{-1}, \omega_{1 e}=\omega_{1 m}=2 \pi \times 5 \times 10^{6} \mathrm{~s}^{-1}$, and include small losses $\gamma_{e}=2 \times \gamma_{m}=2 \pi \times 6 \times 10^{6} \mathrm{~s}^{-1}$. With these parameters, the refractive index of the LH material $n \approx-1$ at frequency $f_{0}=15 \mathrm{GHz}$, and the material is left handed for frequencies $f<18.5 \mathrm{GHz}$ and right handed for $f>26 \mathrm{GHz}$. The RH layer is assumed to be air.

The structure shown in Fig. 1(a) consists of three stacks with four LH/RH double layers each. The outer stacks (stack 1 and stack 3) have individual layer thicknesses of $\lambda_{0} / 5$, while the central stack (stack 2) has individual layer thickness $\lambda_{0} / 3 ; \lambda_{0}$ being the free-space wavelength of incoming

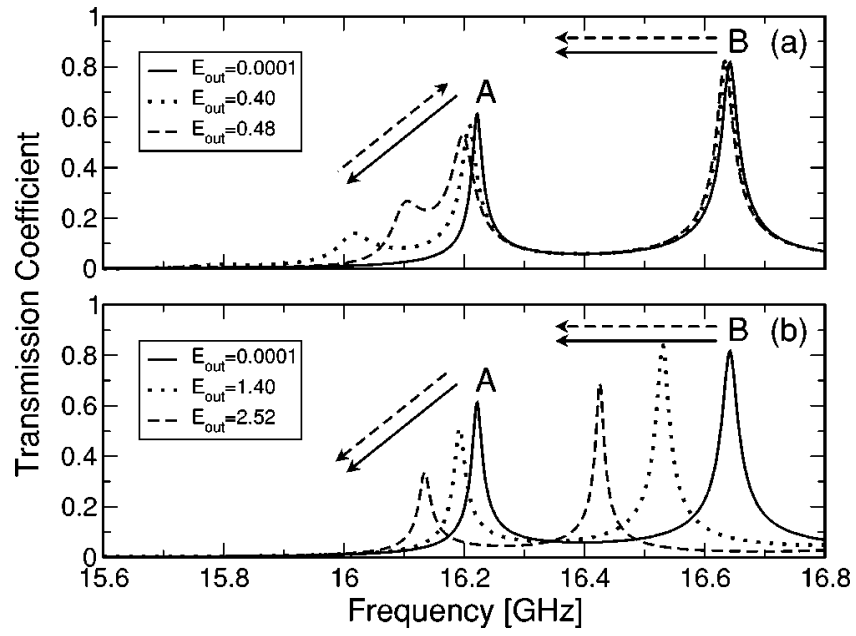

FIG. 3. Transmission spectrum of the structure in Fig. 1(b) for incidence from the (a) left and (b) right, for different output amplitudes, calculated by TMM. The arrows indicate the direction in which the peaks shift with increased input (solid) and output (dashed) intensity.

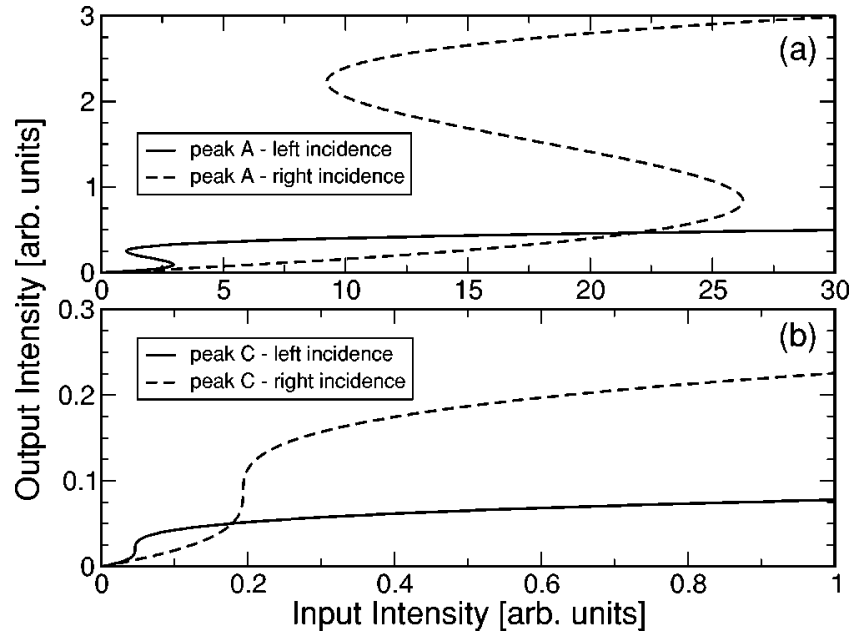

FIG. 4. TMM results: Bistability curves for $f=16.19$ (a) and 25.17 (b) GHz, at equal relative detuning below linear transmission peaks $A$ and $C$. The curves show incidence from the left (solid) and the right (dashed). Peak $C$ shows bistability for greater but not for smaller detuning than shown in (b). Peak $A$ shows bistability even for much smaller relative detuning.

radiation of the frequency $f_{0}$. Figure $1(\mathrm{~b})$ shows the same system with a structural defect introduced between stack 1 and stack 2 . The defect layer has thickness $2 \lambda_{0} / 25$.

First, we study the transmission properties of the individual components of our superlattice in order to analyze the effect of the layer thickness on the positions of the band gaps and the transmission spectrum. Figure 2(a) shows the transmission spectrum of a structure made of the combination of stack 1 and stack 3 directly attached to each other (solid), as well as the transmission spectrum of stack 2 (dashed). The spectra show several transmission bands and gaps, and we clearly see a shift of the conventional band gaps due to the scaling of the structure. However, the band gap associated with vanishing average refractive index $\langle n\rangle=0$ (around

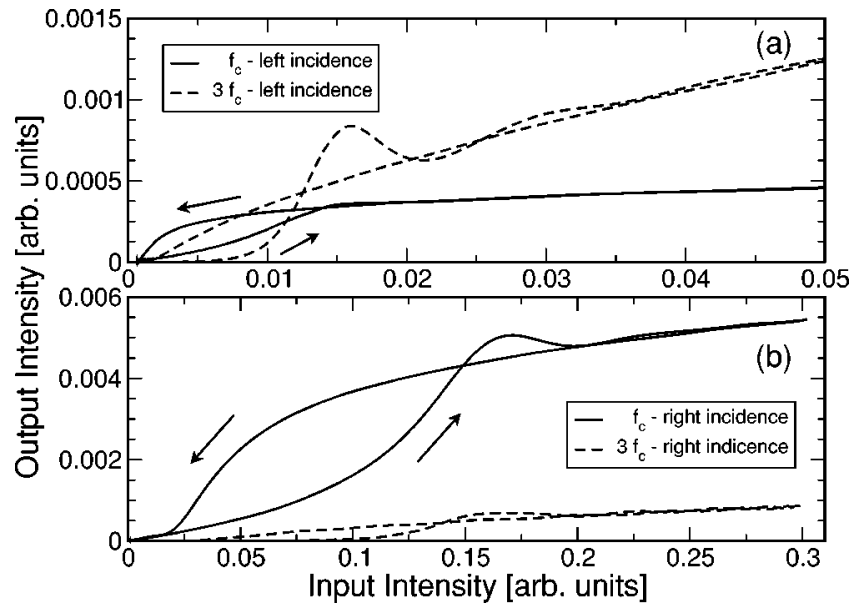

FIG. 5. PSTD results: Hysteresis curve of the structure for a Gaussian pulse incident from the left (a) and the right (b). The incident field has carrier frequency $f_{c}=16.19 \mathrm{GHz}$ (peak $A$ ), width parameter $1100 / f_{c}$, and peak amplitude 0.55 . The arrows indicate the direction of change with time. 
$\left.f_{0}=15 \mathrm{GHz}\right)$ remains at the same frequency and only changes its width [10].

The transmission spectrum of the combined structure of Fig. 1(a) is shown in Fig. 2(b). In the frequency intervals where the central stack is opaque, the structure has a band gap, while at frequencies where the outer stacks are opaque but the central stack is transparent, the structure behaves similar to a cavity resonator and shows the characteristic Fabry-Pérot cavity peaks. In this system cavitylike behavior occurs in the frequency ranges of 13.5-14.3, 16.3-17.5, and $25.8-29.9 \mathrm{GHz}$.

To study the nonlinear transmission of our multistack structure, we introduce a defect layer with a linear electric permittivity $\varepsilon_{r}=4$ [see Fig. 1(b)]. For the TMM calculations, we place a $\delta$-function Kerr-type nonlinearity at the left interface of the defect layer [14] $\varepsilon_{r}\left[1+\delta\left(x-x_{0}\right)\right], x_{0}=1.6 \lambda_{0}$, while in the PSTD calculations the entire defect layer has a nonlinear electric permittivity $\varepsilon_{r}(t)=4+\chi^{(3)}|\mathbf{E}(t)|^{2}$, with the nonlinearity coefficient $\chi^{(3)}=+4$. We study the difference in transmission when the light is incident from opposite sides of the structure. In the linear case, the reciprocity principle holds as expected and the transmission coefficient is independent of direction of incidence, while a nonlinear asymmetric structure can show directional dependence of the transmission indicating the corresponding diode action effect $[1,2]$.

First, we employ the TMM and study the steady-state results. In this situation it is convenient to fix the transmitted (output) field and calculate backward to find the incident and reflected fields. In Fig. 3 we present a spectral interval including the first two peaks (peaks $A$ and $B$ ) above the $\langle n\rangle$ $=0$ band gap of stack 2 for several fixed transmitted field strengths and different directions of incidence. The inclusion of the defect shifts all peaks in Fig. 2(b) to slightly lower frequency in the linear regime. Due to the nonlinear properties of the defect, the frequencies of the transmission resonances vary with the local electric field intensity which in turn varies with the output field. Since the behavior of peak $B$ is very similar for the two directions of incidence, the output field strengths in the figure are chosen to highlight the behavior of peak $A$. The frequency shift of peak $A$ occurs at very different output field strengths depending on the direction of incidence. As the input field is increased, all the transmission peaks shift to lower frequencies (solid arrows in Fig. 3 ). The curves are presented with fixed output field and one notices that all peaks shift to lower frequencies with increased output field (dashed arrows in Fig. 3), except for peak $A$ in the case of incidence from the left [Fig. 3(a)], which moves to higher frequencies. This opposite shift in frequency means that while generally the output field at the transmission peak increases with increased input field, for this peak the output field actually decreases as the input field is increased. Referring to Fig. 2(b) one notices that peak $A$ is located slightly above a band gap of the central stack (stack 2). With increased input intensity the nonlinear defect shifts the peak to lower frequency such that it moves deeper into the band gap and becomes increasingly suppressed. Behavior similar to that of peak $A$ is also found for the first peak (peak $C)$ above the first Bragg band gap of stack 2 (not shown).

Next, in Fig. 4, we compare the output intensity as a function of input intensity for incidence from the two opposite sides at frequencies with equal relative detuning from peaks $A$ and $C$, calculated using the TMM. We find strong nonreciprocity in all cases. The transmission in the two directions differs by up to a factor of 6 for peak $A$ and a factor of 4 for peak $C$. Peak $A$ also shows strong bistability for both directions. The switching intensity threshold for incidence from the left is almost an order of magnitude lower than for incidence from the right. With the given detuning from the linear transmission peak frequency one finds no bistability for peak $C$ but with stronger detuning bistability does appear. Such bistability in an optical diode was previously mentioned in $[2,18]$.

Finally, we simulate the transmission using the PSTD method. This time-domain method inherently includes the higher harmonics that may occur due to the nonlinear material. We use a spatial step size of $\Delta_{x}=\lambda_{0} / 75$ and a temporal step size of $\Delta_{t}=\Delta_{x} /(\pi c)$. The incident field was chosen as a long Gaussian pulse with peak amplitude 0.55 , carrier frequency $f_{c}=16.19 \mathrm{GHz}$ (peak $A$ ), and width parameter $1100 / f_{c}$, to operate in a regime where the envelope magnitude varies slowly. Figure 5 shows the magnitude of the short-time Fourier transform at the carrier frequency and at its third harmonic. Again, we find that the transmission strongly differs for incidence from the two sides. Transmission at $f_{c}$ is initially higher for incidence from the left but when the incident intensity reaches 0.04 transmission for incidence from the right becomes greater. On the other hand, the transmission at $3 f_{c}$ is always much greater for the pulse incident from the left. At $3 f_{c}$ the structure is essentially transparent, as the LH-material slabs have $\varepsilon_{r} \approx \mu_{r} \approx n \approx 1$ at that frequency. We find bistability for the carrier frequency, as well as its third harmonic with incidence from either direction. The threshold switching intensities differ greatly for the two directions. The switching intensities for the two frequencies with a particular direction of incidence are very similar to one another. These results agree well with the TMM calculation.

In our PSTD simulations we also encountered modulational-instability-like behavior in this system with different threshold intensities for the onset of this instability. This effect will be subject to further investigation.

In conclusion, we have studied numerically the linear and nonlinear transmission of a multilayer structure composed of two different dielectric materials. We have shown that asymmetric periodic structures with nonlinear layers can demonstrate highly nonreciprocal transmission, which is a major characteristic of an optical diode. We have analyzed the properties of the left-handed diode by employing the transfer-matrix approach and direct pseudospectral timedomain numerical simulations, and have shown its bistable behavior. 
[1] M. Scalora, J. P. Dowling, C. M. Bowden, and M. J. Bloemer, J. Appl. Phys. 76, 2023 (1994).

[2] M. D. Tocci, M. J. Bloemer, M. Scalora, J. P. Dowling, and C. M. Bowden, Appl. Phys. Lett. 66, 2324 (1995).

[3] J. J. Liang, S. T. Lau, M. H. Leary, and J. M. Ballantyne, Appl. Phys. Lett. 70, 1192 (1997).

[4] K. Gallo and G. Assanto, J. Opt. Soc. Am. B 16, 267 (1999).

[5] S. Mujumdar and H. Ramachandran, Opt. Lett. 26, 929 (2001).

[6] C. B. E. Gawith, P. Hua, P. G. R. Smith, and G. Cook, Appl. Phys. Lett. 78, 4106 (2001).

[7] K. Gallo, G. Assanto, K. R. Parameswaran, and M. M. Fejer, Appl. Phys. Lett. 79, 314 (2001).

[8] See Opt. Express 11, 639 (2003), focus issue: Negative Refraction and Metamaterials.

[9] I. S. Nefedov and S. A. Tretyakov, Phys. Rev. E 66, 036611
(2002).

[10] J. Li, L. Zhou, C. T. Chan, and P. Sheng, Phys. Rev. Lett. 90, 083901 (2003).

[11] M. W. Feise, I. V. Shadrivov, and Yu. S. Kivshar, Appl. Phys. Lett. 85, 1451 (2004).

[12] P. Yeh, Optical Waves in Layered Media (John Wiley \& Sons, New York, 1988).

[13] Q. H. Liu, Microwave Opt. Technol. Lett. 15, 158 (1997).

[14] E. Lidorikis, K. Busch, Q. M. Li, C. T. Chan, and C. M. Soukoulis, Physica D 113, 346 (1998).

[15] J. P. Berenger, J. Comput. Phys. 114, 185 (1994).

[16] M. W. Feise, J. B. Schneider, and P. J. Bevelacqua, IEEE Trans. Antennas Propag. 52, 2955 (2004).

[17] P. Tran, Opt. Lett. 21, 1138 (1996).

[18] L. X. Chen, D. Kim, Y. L. Song, W. Q. Ding, W. H. Lu, and S. T. Liu, Chin. Phys. Lett. 20, 1514 (2003). 